\title{
Psychodynamic approaches to teaching medical students about the doctor-patient relationship: randomised controlled trial
}

\author{
Jessica Yakeley, ${ }^{1}$ Peter Shoenberg, ${ }^{2}$ Richard Morris, ${ }^{3}$ David Sturgeon, ${ }^{3}$ Sarah Majid ${ }^{1,2}$
}

The Psychiatrist (2011), 35, 308-313, doi: 10.1192/pb.bp.110.033704

${ }^{1}$ Tavistock and Portman NHS Foundation Trust; ${ }^{2}$ Camden Psychodynamic Psychotherapy Service, Camden and Islington NHS Foundation Trust; ${ }^{3}$ University College London

Correspondence to Jessica Yakeley (jyakeley@tavi-port.nhs.uk)

First received 1 Dec 2010, accepted 11 Feb 2011

\begin{abstract}
Aims and method To evaluate the effectiveness of two psychodynamic psychotherapy teaching methods, a student psychotherapy scheme (SPS) and participation in a Balint group, in teaching first-year clinical medical students about doctor-patient communication and the doctor-patient relationship. The 28 students, who were randomly allocated to three groups (SPS group, Balint group starting at baseline and Balint group starting at 3 months and acting as partial controls), were rated on a questionnaire testing their knowledge of emotional and psychodynamic aspects of the doctor-patient relationship administered at baseline, at 3 months and at 1 year.
\end{abstract}

Results At 3 months, students in the SPS and Balint groups scored higher than the partial control group, the difference approaching significance at the $5 \%$ level. At 1 year, participation in either teaching method led to significantly higher scores compared with baseline.

Clinical implications Psychodynamic psychotherapy teaching methods are effective in increasing students' knowledge of the doctor-patient relationship and potentially also improving their communication skills.

\section{Declaration of interest None.}

Good communication between a doctor and his or her patient serves two essential functions: to share clinical information and to establish a good doctor-patient relationship. ${ }^{1}$ Effective doctor-patient communication is associated with many benefits, which include patient satisfaction, adherence to treatment, symptom improvement, reduction in psychological distress, and patient's perception of physician competence. ${ }^{2}$ Moreover, the majority of complaints and lawsuits against doctors cite poor communication as the main cause of the patient's grievance. ${ }^{3}$ These findings have led to the prioritisation of teaching communication skills in medical school curricula in recent years, yet a number of issues still need to be considered. There is disagreement about what the most effective way of teaching communication skills is, but there is evidence that more experiential methods of learning are preferred by students and are more effective than didactic demonstrating or lecturing. ${ }^{4}$ It is also not clear at what stage in their training and for what duration students should be taught communication skills, and whether different students are suited to different teaching methods. ${ }^{5,6}$ Many programmes now use actors in simulated doctor-patient consultation scenarios and provide video feedback of the student's interviewing skills, but it is not clear how well these acquired skills translate to real encounters with patients. Many of the studies attempting to evaluate different methods for teaching communication skills lack a control group, ${ }^{7}$ and often the main measurement is based on the student's own self-evaluation, which does not correlate with other measures, such as evaluation by their teachers, and may not be indicative of actual improvement as poorly performing students tend to overrate their ability. ${ }^{8,9}$

\section{Psychodynamic teaching at University College London School of Medicine}

We were interested in evaluating the effectiveness of two methods of teaching medical students about communication between doctor and patient and about the doctorpatient relationship: the University College London (UCL) student psychotherapy scheme (SPS) and Balint groups, both of which are based on a psychodynamic paradigm and are focused on increasing students' awareness and understanding of the emotional aspects of the doctor-patient relationship.

At the beginning of each academic year all of the firstyear clinical medical students on the introductory course are invited to an introductory lecture about the SPS and Balint groups: interested students then volunteer to join. Each year, about $30-40 \%$ of the annual student intake 
express an interest in participating in either one or both of these groups, and are then interviewed by one of the senior members of the psychotherapy department to determine their suitability, mainly for their potential participation in the SPS (because it will involve them in seeing a patient for psychodynamic psychotherapy). Suitability is determined by the degree to which the student appears to understand what the scheme will involve and their motivation to make what in effect is a very large commitment of their private time to this extra learning activity. We also want to ensure that the student is not currently experiencing personal difficulties, for example a recent bereavement, which could interfere with their ability to be a student therapist. Each year, about $95 \%$ of student applications are considered 'suitable' and about $5 \%$ 'not suitable'.

\section{Student psychotherapy scheme}

The SPS has now been running at UCL for over half a century, having been initiated in 1958 in the then Outpatient Department of Psychological Medicine in University College Hospital. ${ }^{10,11}$ It has continued to operate under the aegis of UCL School of Medicine, and is offered to first-year clinical medical students in the Camden Psychodynamic Psychotherapy Service (Camden and Islington NHS Foundation Trust). This scheme helps medical students to learn communication skills and about the doctor-patient relationship in greater depth, by allowing them to see a carefully selected out-patient for once-weekly psychodynamic psychotherapy for 1 year, in a small group, under weekly supervision from one of the senior members of the department. ${ }^{12-16}$ All the patients seen by the students have consented to a student delivering their psychodynamic psychotherapy. This experience has proved helpful both to patients as an important introduction to psychotherapy and to students in providing them with a prolonged psychodynamic contact with a patient.

\section{Balint groups}

Each year many students are disappointed that they cannot get a place on the scheme, as we can only offer places to a very limited number (usually 10-15 students) per year, given the considerable time commitment it demands from senior clinical staff and the difficulties in finding suitable patients for the students to see. To meet student demand, in 2004 we initiated Balint groups ${ }^{17}$ as another form of teaching about the doctor-patient relationship that could be available to a larger number of medical students. These are weekly discussion groups, based on the ideas of Michael Balint, who originally developed these groups with general practitioners. Students are encouraged to talk about their clinical experiences with patients, to explore the emotional aspects of medical illness and to learn more about the relationship of the student/doctor to the patient. Each group comprises ten medical students and is facilitated by two specialist leaders, one a medically qualified psychotherapist, usually a consultant psychiatrist or specialist registrar in psychotherapy, and the other a general practitioner who is qualified as a Balint group leader. The groups meet weekly for 12 weeks. The students are encouraged to talk spontaneously about patients with whom they have had direct contact and who have interested, intrigued, puzzled or upset them. Discussions are facilitated about the emotional aspects of medical illnesses, and how a student's or a doctor's responses to the patient can be informative about the patient's pathology. We can currently offer 10 groups every year, accommodating over 100 students. They are now a recognised part of the curriculum and are offered to the first-year clinical students as a student selected component.

Both the SPS and Balint groups continue to be very popular and oversubscribed, and many of the students tell us that this offers them a unique experience in being able to learn about the doctor-patient relationship in a way that had not previously been available to them. As well as receiving the students' enthusiastic and positive feedback, we wanted to demonstrate empirically that these two teaching interventions were effective in improving the students' communication skills, specifically their awareness, knowledge and understanding of the emotional aspects of the doctor-patient relationship.

\section{Study design and background}

This study is a randomised controlled trial comparing three groups of students over the period of 1 year: group 1 , students who participated in the SPS; group 2, students who participated in a Balint group; and group 3, a partial control group composed of students who were waiting to participate in a Balint group at 3 months and so had not yet been subjected to either method at this time. Our working hypothesis was that both psychotherapy teaching approaches would lead to a better understanding of the doctor-patient relationship compared with students in the control group who had participated in neither teaching experience at 3 months.

\section{Method}

\section{Participant selection}

The research sample were medical students starting their first year of clinical medicine in September 2006. Following the introductory lecture, at which we also explained the nature and purpose of our research, 49 students volunteered for both psychotherapy and Balint group options and were interviewed by one of four senior members of the psychotherapy department. The interviews were semistructured but brief, asking the students why they wanted to join the student psychotherapy scheme, whether they understood the responsibilities involved, and whether they anticipated any difficulties. Of the 49 students, 46 were deemed 'suitable'; the remaining 3 were deemed 'not suitable' because of personal difficulties and were not included in the research, but were offered participation in a separate Balint group that was not part of the research.

Of the 46 students, we randomly selected 30 for the research project. The remaining 16 were offered participation in a Balint group not involved in the research. The students were randomly allocated to one of three groups, containing 10 students each:

- group 1 - students who would participate in the SPS from January 2007 to January 2008 
- group 2 - students who would participate in a Balint group from January 2007 to April 2007

- group 3 - students who would participate in a Balint group from April 2007 to July 2007, and so would act as a partial control for groups 1 and 2 for the first 3 months of the research.

We decided not to have a pure control group, as many of the students volunteering for the research had opted to do the SPS or a Balint group for their student selected component. Both Balint groups were run by the same two group leaders, to avoid any variability due to group leaders' style, which might have interfered with comparisons between the two groups.

All students gave their written consent for participating in the research and understood that their participation was voluntary and that they were free to withdraw at any time, without giving any reason, and without their education or legal rights being affected. The research project was reviewed and approved by the Camden and Islington local research ethics committee.

\section{Questionnaire}

Because we could not find a suitable pre-existing validated measure that tested knowledge of emotional aspects of the doctor-patient relationship, we designed our own measure, a qualitative questionnaire that asked a series of questions about the emotional aspects of the doctor-patient relationship (Appendix). The questionnaire aimed to ascertain that the student had become aware of the significance of the relationship between the doctor/student and patient, that the student recognised the feelings which were evoked by the interaction with the patient and was able to use these for the benefit of the patient, that the student could be aware of the emotional meanings of the patient's physical symptoms, and that the student was aware of his or her own limitations. We sent the questionnaire to the students and asked them to return it after completion in a pre-paid addressed envelope. The questionnaire took up to half an hour to complete.

We administered the questionnaire to all three groups at three points in time: before joining the teaching option (in January 2007), at 3 months after the teaching option had started (April 2007), and at 1 year after the teaching option had started (January 2008). At 3 months, group 3 had not yet participated in either teaching option, and could therefore act as a control to groups 1 and 2. At 1 year, all the groups had participated in one of the two teaching methods.

The questionnaires were marked by three independent raters (D.J., S.M. and D.S.). Because of the layout of the questionnaire (the last question asked the students to comment on their experience of the project), it was not possible to say that the rating would be entirely masked, as one of the raters has commented. However, the other two raters stated that their marking was masked as this potentially identifying question occurred at the end of each questionnaire. The questionnaires were marked according to a list of 'set answers' that we had compiled (see the online supplement to this paper).

\section{Statistical analysis}

Averages of the three raters' scores for each student at each time point were calculated. Comparisons of average scores at 3 months were made among the three groups, with group 1 (SPS) as reference. Adjustments were made for baseline scores using analysis of covariance (ANCOVA). ${ }^{18}$ A similar analysis was carried out on 12-month scores, again adjusting for baseline. As all three groups had received some intervention at 12 months, a paired $t$-test was carried out to assess the degree to which students had changes in mean scores since the baseline assessment; 95\% confidence intervals were calculated for each comparison made.

\section{Results}

Shortly after the research started, two students dropped out of group 1, one due to illness and the other because of time constraints; this left eight students in group 1, ten in group 2 and ten in group 3.

We did not achieve a complete return rate for the questionnaires, and the rate was particularly poor at 3 months. At baseline, 25 of 28 students returned completed questionnaires, at 3 months this had dropped to 15 of 28 , but at 1 year we achieved a return rate of 23 of 28 . We took the mean score of the three raters for each student, and from this calculated the mean score (and standard deviation) for each group at each time point (Table 1).

At 3 months the mean difference in scores between the SPS group and Balint group 1 was negligible (adjusted difference in means $0.1,95 \% \mathrm{CI}-2.5$ to $2.6, P=0.96$ ), but the difference in scores between the SPS group and Balint group 2 approached statistical significance at the $5 \%$ level (adjusted difference in means $-2.2,95 \%$ CI -4.8 to 0.4 , $P=0.083)$. Similarly, the difference in scores between Balint group 1 and Balint group 2 was approaching significance (adjusted difference in means $-2.2,95 \%$ CI -4.6 to 0.3 , $P=0.076$ ). However, this was based on only 14 students at this time, as one student had not returned a questionnaire.

At 1 year, there was no significant difference between any of the three groups, but the mean difference in scores ( $n=21 / 28$ students who filled in questionnaires at both baseline and 1 year) between the start of the project and after 1 year was highly significant (mean difference 1.5, 95\% CI 0.6 to $2.4, P=0.0023$ ).

Recorded scores at all three time points were pooled, giving 62 data points for which comparisons could be made between the three observers. The mean scores for all

\begin{tabular}{|llcc|}
\hline Table 1 & \multicolumn{2}{l}{$\begin{array}{l}\text { Students' scores on the questionnaire at three } \\
\text { time points }\end{array}$} \\
Group & \multicolumn{3}{c|}{ Mean (s.d.) } \\
\cline { 2 - 4 } & Baseline & 3 months & 1 year \\
\hline Student psychotherapy & $\begin{array}{c}6.7(3.0) \\
(n=8)\end{array}$ & $\begin{array}{c}7.7(1.4) \\
(n=4)\end{array}$ & $\begin{array}{c}8.0(3.1) \\
(n=8)\end{array}$ \\
scheme & $\begin{array}{c}6.8(1.2) \\
(n=8)\end{array}$ & $\begin{array}{c}7.6(1.7) \\
(n=5)\end{array}$ & $\begin{array}{c}8.3(1.8) \\
(n=7)\end{array}$ \\
\hline Balint group 1 & $6.0(2.2)$ & $5.4(1.7)$ & $7.4(0.8)$ \\
& $(n=9)$ & $(n=5)$ & $(n=8)$ \\
\hline Balint group 2 & & & \\
& & & \\
\end{tabular}


students at all time points were 6.8, 5.6 and 8.7 respectively, and the within-student standard deviation was 2.4. The correlations between scores of pairs of observers ranged from 0.49 to 0.65 .

\section{Discussion}

The main findings are a significant improvement in the scores compared with baseline in all three groups at 1 year, after the students had all participated in one of the teaching methods, and a trend (that did not quite reach significance) towards higher scores at 3 months in the two groups who had participated in the SPS and Balint groups compared with the control group who had not participated in either intervention at that stage. These results support our hypothesis in suggesting that the two interventions are effective in increasing students' knowledge of the doctorpatient relationship.

Previous studies have shown that students' acquisition of knowledge of communication skills may be optimised when communication training is given with supervised patient contact, ${ }^{19}$ of which both the SPS and Balint groups would be examples. Moreover, a meta-analysis of 24 randomised controlled trials evaluating the effects of teaching interventions on medical students' patient communication skills showed that the two most effective teaching methods in improving student performance were direct feedback on a student-patient interview and small-group discussions. ${ }^{7}$ However, none of the studies in the metaanalysis included student psychotherapy schemes or Balint groups, and we believe our study to be the first reported randomised controlled trial of these teaching methods, both of which revolve around small-group discussion and detailed feedback regarding the student's direct contact with patients, from either the student's supervisor in the case of the SPS or from the Balint group leader.

\section{Limitations}

There were various limitations to our study. First, there was no control group at 1 year, and there was no significant difference at 1 year between the scores of all three groups. We therefore cannot confidently assume that the significant improvements in the students' scores at 1 year compared with their scores at the beginning were due to the effects of the SPS or Balint groups, and not other influences they have been subject to having completed a year of clinical medical studies, such as specific curriculum factors or the frequent observation of clinician-patient contacts. An important component of the medical school curriculum at the UCL School of Medicine is the professional development spine, which was developed in line with the recommendations of Tomorrow's Doctors ${ }^{20}$ and emphasises the importance of patient-centred medicine. There is also a preclinical communication skills teaching component, but this is more behavioural than reflective, focusing on issues such as the value of open $v$. closed questions, eye contact and body language, rather than emotional and relational aspects of communication.

An alternative explanation for the significant increase in scores in all the groups in our study at 1 year could be maturation of the students over time rather than the effect of any teaching programme. However, such a hypothesis is not supported by the literature, which suggests a diminution of communication skills in medical students without some formal input. ${ }^{21}$ One interesting result in our study was the decrease in scores from baseline at 3 months in the partial control group of students who had as yet received no intervention. Other researchers have noted a worrying decline in communication skills, patient-centred attitudes and empathy in medical students as they progress through medical school, which has been attributed to the dominant medical culture promoting biomedical mechanisms of disease rather than psychosocial determinants. ${ }^{22-25}$ Students may lose their idealism and wish to help others, becoming disillusioned and cynical and developing coping mechanisms of distance and detachment at the expense of their awareness of patients' concerns and emotions.

A second limitation of our study was the small number of students involved and the low number of questionnaire returns at 3 months. The numbers of students that we could include in this research project were limited by the number of places that we had available on the SPS, which was originally ten, and we did not anticipate that two of the students would drop out at the beginning. The poor return rate at 3 months may have been caused by students' reluctance to fill out the same questionnaire they had only completed 3 months before.

Another limitation was that we did not use a preexisting validated measure of communication skills, but devised a measure ourselves. Most measures of communication skills require trained raters to assess videotaped encounters between students and standardised patients, which are costly, time consuming, and often have poor interrater reliability. ${ }^{26}$ Empathy and patient-centred attitudes have been identified as key aspects of the doctorpatient relationship. ${ }^{27}$ However, empathy and related concepts such as emotional intelligence, psychological mindedness and reflective function are complex constructs that reflect a range of cognitive, emotional and behavioural phenomena that reflect a person's personality and are difficult to measure. Empathy and emotional intelligence in medical students have been measured in previous studies by instruments such as the Bar-On Emotional Quotient Inventory, ${ }^{28}$ and patient-centred attitudes by the questionnaires such as the Patient-Practitioner Orientation Scale $^{21,23}$ and the Doctor-Patient Communication Inventory. ${ }^{29}$ However, we felt that these existing measures did not address knowledge of the doctor-patient relationship from a psychodynamic viewpoint, that is considering an understanding of the unconscious processes involved in emotional communication or the importance of a patient's prior attachment experiences in influencing their relationship to the doctor. We therefore decided to design our own questionnaire that would meet all those requirements. The questionnaire is, of course, testing students' knowledge at a theoretical level, and so we do not know whether, and how, such acquired knowledge translates into actual behavioural change in the form of improved communication skills with patients. Our ideal answers to the questionnaire may also have assumed a greater potential in the students for learning about the doctor-patient relationship than was 
possible with so short an exposure to these psychodynamic teaching approaches, and may explain why the changes in the three groups were relatively small.

The interrater reliability was not perfect, with the observers having different overall mean scores, and less than ideal correlation between the scores. If the observers had been masked, any biases should not have affected differences seen between groups at 3 months. Since only one person commented that they were not masked when making assessments, the analysis was repeated with their results omitted. Results were essentially unchanged, with magnitudes of differences among groups being very similar to those reported. Indeed, the lack of reliability would have tended to mask true effects of the intervention, so we believe the poorer performance for the control group at 3 months is likely to be real.

\section{Implementing psychotherapy teaching methods on a wider scale - benefits}

The results of our study suggest that psychotherapy teaching and Balint groups could help medical students to learn about the doctor-patient relationship and as such could be useful additions to the undergraduate curriculum. Running a student psychotherapy scheme requires considerable expertise and resources, as well as involving complex clinical governance and ethical issues, and will therefore only be available to a small proportion of students in any year group. A similar scheme has recently been established at the University of Bristol (R. Brown, personal communication, 2009). Balint group teaching also requires experienced group leaders, but is able to accommodate many more students owing to the shorter duration of the course. Balint groups have been used as a teaching method for medical students and have been reported in the literature in several countries, including Germany, ${ }^{30}$ Italy, ${ }^{31-33}$ South Africa, ${ }^{34}$ Poland, ${ }^{35}$ Finland $^{36}$ and the USA. ${ }^{37}$ However, although Balint-style case discussion groups are now a compulsory part of the postgraduate curriculum for junior psychiatrists in the UK, to our knowledge there have been no published reports of Balint groups for medical students in the UK since Balint himself experimented with medical student discussion groups at UCL. ${ }^{27}$

Although the aim of both interventions is to teach the student about the doctor-patient relationship, inevitably the students doing the SPS will learn a lot about psychodynamic psychotherapy, whereas in the SPS and the Balint groups the students will have contact with psychiatrists supervising their patients or running the Balint group. In a retrospective study of the career choices of 200 students who did the SPS between 1982 and 1992 and who were compared with 200 randomly selected control students in the same period who did not do this scheme, we found that the students who participated in the SPS, even if they were not originally planning a career in psychiatry, were significantly more likely to choose psychiatry as a career than those who did not do the SPS. ${ }^{16}$ This finding is important given the current recruitment crisis in psychiatry. As the Balint groups at UCL have only been running for the past 6 years, we have not had a chance as yet to follow up these students' career pathways. Nevertheless, we might hypothesise that the experience of participating in a Balint group, in which the students are exposed to thinking about psychological issues in an innovative way, might foster the students' curiosity in considering psychiatry as their potential chosen specialty. In the meantime, we are encouraged that we are oversubscribed every year thanks to the immense popularity of both of these teaching opportunities, which we hope to develop further.

\section{Acknowledgement}

We would like to thank Dick Joyce for his contribution to the rating and for many very helpful discussions during the preparation of this paper.

\section{Appendix}

\section{Questionnaire about the doctor-patient relationship}

1 What effect can the relationship between a doctor/ student and patient have on the patient's overall care?

2 How may a doctor's/student's feelings be affected by a patient?

3 How may a doctor/student use those feelings in relation to the patient?

4 How do you cope with your anxiety and uncertainty in your work with patients?

5 Do you feel that the relationship between the doctor/ student and the patient should be an equal one? If not, why?

6 Why is it important to understand the nature of the patient's attachment to the doctor/student?

7 How do you recognise emotion in a patient when it is not verbalised?

8 Please comment on your experience in this project.

\section{About the authors}

Jessica Yakeley is Consultant Psychiatrist in Forensic Psychotherapy at the Portman Clinic, and Director of Medical Education and Associate Medical Director, Tavistock and Portman NHS Foundation Trust. Between 2003 and 2010 she was Consultant Psychiatrist in Psychotherapy in the Camden Psychodynamic Psychotherapy Service (formally University College London (UCL) Hospital and the Royal Free Departments of Psychotherapy). Peter Shoenberg is Consultant Psychiatrist in Psychotherapy at the Camden Psychodynamic Psychotherapy Service, Camden and Islington NHS Foundation Trust, and Head of the UCL Student Psychotherapy Scheme. Richard Morris is Professor of Medical Statistics and Epidemiology in the Department of Primary Care and Population Health at UCL, and from 2000 to 2008 led the 'Evaluation of Evidence' component of the Professional Development Spine in UCL's medical undergraduate curriculum. David Sturgeon is Consultant Psychiatrist at the Student Psychological Therapies Service, UCL. He was formerly Consultant Liaison Psychiatrist, UCL Hospital. Sarah Majid is a Consultant Psychiatrist in Psychotherapy working in Camden Psychotherapy Service, Camden and Islington NHS Foundation Trust, and in the Adult Department, Tavistock and Portman NHS Foundation Trust.

\section{References}

1 St Claire L. Measuring communication skills of medical students to patients with cancer. Br J Med Psychol 2001; 73: 99-116.

2 Shapiro SM, Lancee WJ, Richards-Bentley CM. Evaluation of communication skills program for first-year medical students at the University of Toronto. BMC Med Educ 2009; 9: 11. 
3 Tamblyn R, Abrahamowicz M, Dauphinee D, Wenghofer E, Jacques A, Klass $D$, et al. Physician scores on a national clinical skills examination as predictors of complaints to medical regulatory authorities. JAMA 2007; 298: 993-1001.

4 Rees CE, Sheard CE. The reliability of assessment criteria for undergraduate medical students' communication skills portfolios: the Nottingham experience. Med Educ 2004; 38: 138-44.

5 Aspegren K, Lonberg-Madsen P. Which basic communication skills in medicine are learnt spontaneously and which need to be taught? Med Teach 2005; 27: 539-43.

6 Deveugle M, Derese A, De Maesschalck S, Willems S, Van Driel M, De Maeseneer J. Teaching communication skills to medical students, a challenge in the curriculum? Patient Educ Couns 2005; 58: 265-70.

7 Smith S, Hanson JL, Tewksbury LR, Christy C, Talib NJ, Harris MA, et al. Teaching patient communication skills to medical students: a review of randomized controlled trials. Eval Health Prof 2007; 30: 3-21.

8 Chur-Hanson A. The self-evaluation of medical communication skills. Higher Educ Res Devel 2001; 20: 71-9.

9 Woolliscroft JO, TenHaken J, Smith J, Calhoun JG. Medical students' clinical self-assessments: comparisons with external measures of performance and the students' self-assessments of overall performance and effort. Acad Med 1993; 68: 285-94.

10 Ball $\mathrm{DH}$, Wolff $\mathrm{HH}$. An experiment in the teaching of psychotherapy to medical students. Lancet 1963; 1: 214-7.

11 Sturgeon D. Development and organisation of the studentpsychotherapy teaching scheme at University College Hospital. In First Steps in Psychotherapy (eds HH Wolff, W Knauss, W Brautigam): 28-33. Springer-Verlag, 1983

12 Garner P. Psychotherapy: experiences of medical students. BMJ 1981; 280: 797-8.

13 Clifford GJ. A medical student's experience of dynamic psychotherapy with a young woman suffering from asthma. In Proceedings of the 15th European Conference on Psychosomatic Research (eds JH Lacey, DA Sturgeon): 366-8. John Libbey, 1986.

14 Shoenberg PJ. The Student Psychotherapy Scheme at the University College and Middlesex School of Medicine: its role in helping medical students to learn about the doctor/patient relationship. J Balint Soc 1992; 20: 10-4.

15 Hoy L. Personal view: it's good to talk. BMJ 2002; 324: 57.

16 Yakeley JW, Shoenberg P, Heady A. Who wants to do psychiatry? The influence of a student psychotherapy scheme - a 10-year retrospective study. Psychiatr Bull 2004; 28: 208-12.

17 Shoenberg P, Suckling H. A Balint Group for Medical Students at Royal Free and University College School of Medicine. J Balint Soc 2004; 32 20-3.

18 Frison L, Pocock SJ. Repeated measures in clinical trials: analysis using mean summary statistics and its implications for design. Stat Med 1992; 11: $1685-704$

19 Baerheim A, Hjortdahl P, Holen A, Anvik T, Fasmer OB, Grimsted H, et al. Curriculum factors influencing knowledge of communication skills among medical students. BMC Med Educ 2007; 7: 35.
20 General Medical Council. Tomorrow's Doctors: Recommendations on Undergraduate Medical Education. GMC, 2002.

21 Humphris GM, Kaney S. Assessing the development of communication skills in undergraduate medical students. Med Educ 2001; 35: 225-31.

22 Haidet P, Dains JE, Paterniti DA, Hechtel L, Chang T, Tseng E, et al. Medical student attitudes toward the doctor-patient relationship. Med Educ 2002; 36: 568-74.

23 Nogueira-Martins CMF, Nogueira-Martins LA, Turato ER. Medical students' perceptions of their learning about the doctor-patient relationship: a qualitative study. Med Educ 2006; 40: 322-8.

24 Tsimtsiou Z, Kerasidou O, Efstathiou N, Papaharitou, S, Hatzimouratidis K, Hatzichristou D. Medical students' attitudes to patient-centred care: a longitudinal survey. Med Educ 2007; 41: 146-53.

25 Shapiro J. Walking a mile in their patients' shoes: empathy and othering in medical students' education. Phil Ethics Humanities Med 2008; 3: 10.

26 Lurie SJ, Mooney CJ, Nofziger AC, Meldrum SC, Epstein RM. Furter challenges in measuring communication skills: accounting for actor effects in standardized patient assessments. Med Educ 2008; 42: 662-8.

27 Balint M, Ball DH, Hare, ML. Training medical students in patientcentred medicine. Compr Psychiatry 1969; 10: 249-58.

28 Fletcher I, Leadbetter P, Curran A, O'Sullivan H. A pilot study assessing emotional intelligence training and communication skills with 3rd year medical students. Patient Educ Couns 2009; 76: 376-9.

29 Schneider DE, Tucker RK. Measuring communicative satisfaction in doctor-patient relations: the Doctor-Patient Communication Inventory. Health Communication 1992; 4: 19-28.

30 Sollner W, Maurer G, Mark-Stemberger B, Wesiack W. Characteristics and problems of Balint groups with medical students. Psychother Psychosom Med Psychol 1992; 42: 302-7.

31 Castiglioni R, Bellini M. The psychological training of medical students using Balint's method: analysis of motivations for the group experience. Medicina Psicosomática 1982; 27: 411-9.

32 Luban-Plozza B. A new training method - 20 years of student Balint groups. Schweiz Rundsch Med Praxis 1989; 78: 1192-6.

33 Luban-Plozza B. Empowerment techniques: from doctor-centred (Balint approach) to patient-centred discussion groups. Patient Educ Couns 1995; 26: 257-63.

34 Levenstein S. An undergraduate Balint group in Cape Town. S African Med J 1980; 62: 89-90.

35 Jugowar B, Skommer M. The effectiveness of Balint training for medical students. In The Doctor, the Patient and their Wellbeing - World Wide Proceedings of the 13th International Balint Congress (eds J Salinsky, H Owen): 104-8. Ruckzuckdruck, 2003.

36 Torppa MA, Makkonen E, Martenson C, Pitkala KH. A qualitative analysis of student Balint groups in medical education: contexts and triggers of case presentations and discussion themes. Patient Educ Couns 2008; 72: 5-11.

37 Brazeau CMLR, Boyd L, Rovi S, Tesar CM. A one year experience in the use of Balint groups with third year medical students. Fam Syst Health 1998; 16: 431-6. 